\title{
Analysis of the cutting moments for the selected chopper's cutting drums constructions
}

\author{
Marcin Zastempowski ${ }^{1 *}$ and Andrzej Bochat $^{1}$ \\ ${ }^{1}$ UTP University of Science and Technology in Bydgoszcz, Faculty of Mechanical Engineering, Al. prof. S. Kaliskiego 7, 85-796 \\ Bydgoszcz, Poland
}

\begin{abstract}
The analysis of the drum cutting assembly's movement dynamics is presented in the article. The derived mathematical dependences describing the dynamic load, may be used at the stage of designing of these types of cutting assemblies and of their drive systems. The simulation calculations conducted on the derived mathematical dependences, allowed the authors to develop a computational tool for quick identification of the demand for power and forces operating in the drum cutting assemblies under the impact of changes in characteristic input quantities.
\end{abstract}

\section{Introduction}

The drum cutting assembly is the basic working assembly of a chaff cutter, the task of which is to cut the plant material (stalks or blades) into parts of a determined length, that is called chaff. Application of a cutting assembly of that type, ensures a required degree of material's shredding, what makes it possible to use it for power purposes (biomass) or for nutritional purposes for animals.

In fig. 1, a selected example of a machine is presented, the basic working assembly of which is the drum cutting assembly.

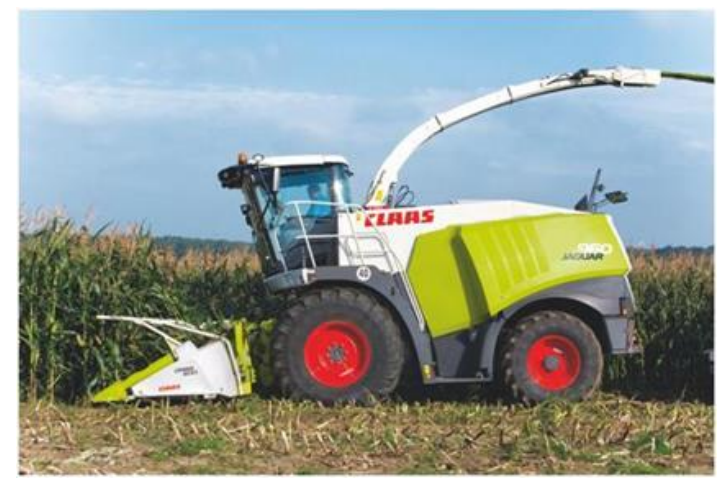

Fig. 1. Self-propelled chaff cutter of Claas [12].

\section{Construction of the drum cutting assemblies and the rules of their operation}

The exemplary construction of a drum cutting assembly is presented in fig. 2. The cutting drums may be of an open or a closed construction. A drum of an open construction consists of a shaft, on which shields with openings are mounted. Cutter holders are fastened to the shields. Cutting knives are fastened in the cutter holders. Depending on the drum's construction, the knives may be straight or bended along the screw line.

In the cutting drum of a closed design, a construction in the form of a closed roller is fastened on a shaft, on the side surface of which brackets are arranged with cutting knives attached to them.

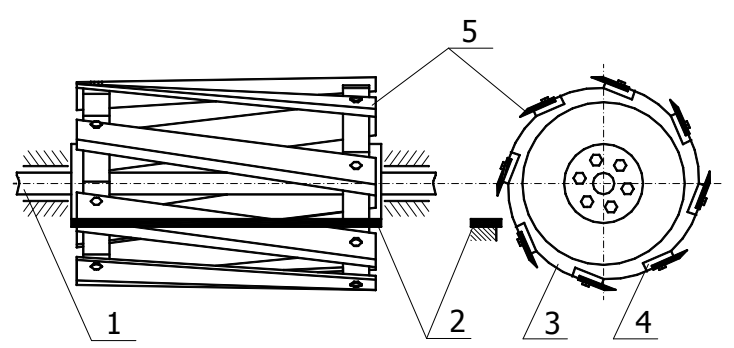

Fig. 2. Cutting drum of a chaff cutter: 1- shaft of the cutting drum, 2 - crosscut edge called a shear bar, 3 - shield of a cutting drum, 4 - cutter holder, 5 - cutting knife.

Rotational motion of a cutting knife causes translocating with that of also cutting knives, what, as an effect, cuts the layer of plant material on the crosscut edge.

Supplying material to the cutting zone takes place through the rotational motion of feeding-crushing rollers, which preliminarily form and compact the material. The essence of the process of plant material's supplying to the cutting drum, is presented in fig. 3 .

In spite of big popularization of agricultural chaff cutters equipped with drum cutting assemblies,

* Corresponding author: zastemp@utp.edu.pl 
constructions of their cutting assemblies have not been changed lately.

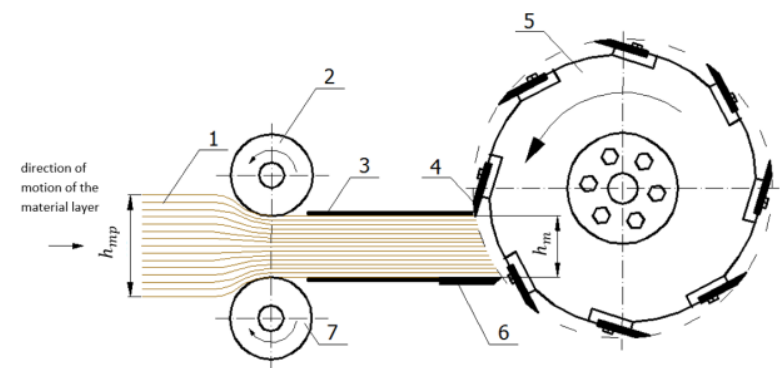

Fig. 3. Process of plant material's supply to the cutting drum [1]: 1 - material's layer, 2 - upper intake-crushing roller, 3 - pressure plate, 4 - cutting knife, 5 - cutting drum, 6 - shear bar, 7 - lower intake-crushing roller, $h_{m p}$ - height of the material's layer prior to compacting, $h_{m}$ - height of the material's layer following compacting, subject to cutting.

From the balance of power (Sankey'a) consumed by a chaff cutter with a drum cutting assembly it results, that the power consumed by a cutting assembly clearly dominates over the powers consumed by the remaining working assemblies, and amounts to $75-80 \%$ [1]. The additionally existing design solutions of cutting assemblies are characterised by high energyconsumption of the cutting process, and what is connected with that, as an effect - their drive systems are equipped with engines of high powers. It is shown by the fact, that the known design solutions have been developed to a big degree on the basis of a construction's intuition. It is connected with the lack of a detailed analysis of drum cutting assemblies' motion dynamics and with the lack of mathematical models describing their cutting process on the basis of which simulation calculations, optimization of construction and improvement of functioning performance, may be conducted [2]. Based on the analysis of the available literature it may be established, that other authors, within the frames of machines' constructions, have mainly taken up the issues connected with the designing rules and analysis of a construction's strength [3], with the rules of use of MES and numerical analysis [4, 5] with mathematical modelling and a construction's optimization [6-9] and the impact of technical devices on environment [10].
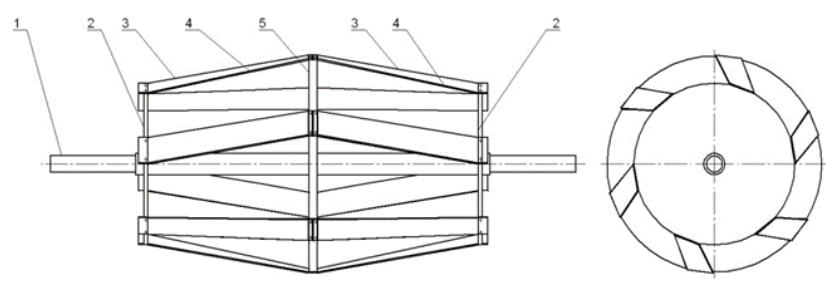

Fig. 4. New construction of the cutting drum [own study]: $1-$ shaft, 2 - external shields, 3 - knives, 4 - cutting edges, 5 central shield.

Looking for more power effective design solutions of the drum cutting assembly performing cutting of plant material into chaff at the Working Machines Institute of
WIM UTP, a new design of the cutting assembly has been developed, patented and performed, which allow for cutting material in a skew -slant manner, the effect of which is a considerable lowering of energy consumption of the cutting assembly's work (fig. 4) [11].

\section{Selected simulation calculations}

Within the frames of the drum movement dynamic's analysis of the cutting assembly, the authors have developed the cutting process's mathematical models on the basis of which numerical calculations have been conducted for real design and exploitation parameters of the cutting assembly. The calculations have been conducted in the BĘBEN_1 computer programme developed by the study's authors. The exemplary programme's window is presented in fig. 5 .

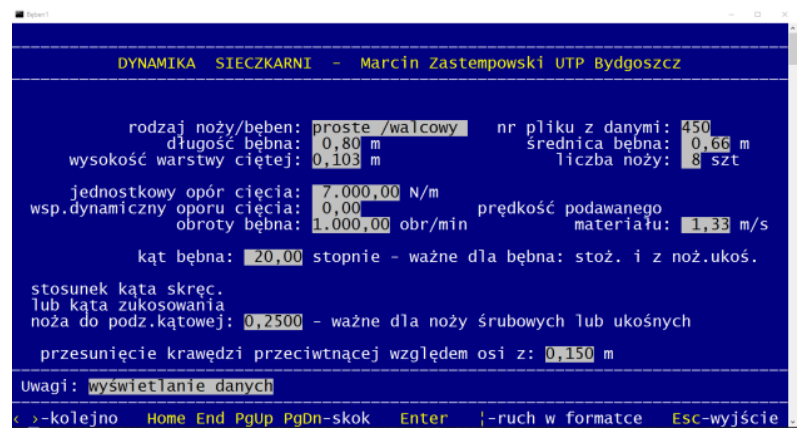

Fig. 5. View of the programme's window [own study].

The calculations have been conducted for five different drum constructions of cutting assemblies. Below there are presented the exemplary results from calculations for a drum of a conical construction with screw knives. In fig. 6 there are presented the geometrical parameters of a cutting assembly for which the calculations have been conducted.

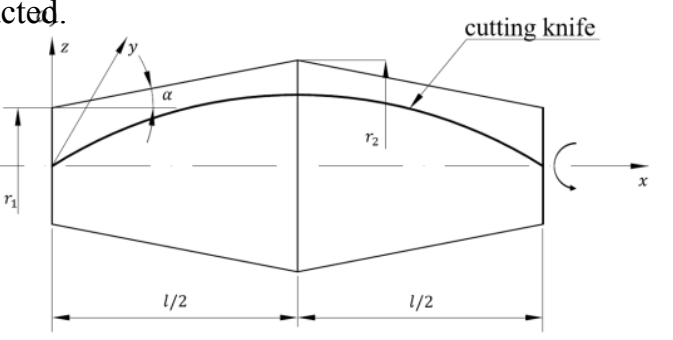

b)

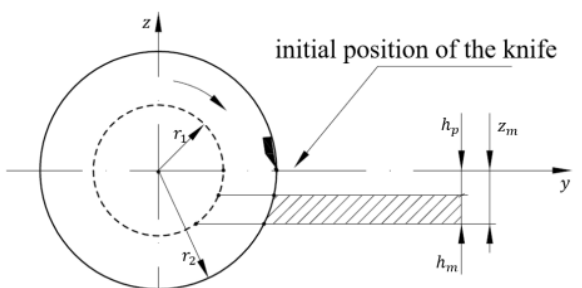

Fig. 6. Geometrical parameters of a drum cutting assembly of a conical type with screw knives [own study]: a - drum in view, b - drum's section for a coordinate $x=l / 2, r_{1}, r_{2}$ - minimum and maximum drum's radius, $\alpha-$ drum's angle (cone's angle of inclination), $l$ - total drum's length, $z_{m}$ - distance of the drum's axis from the crosscut edge, $h_{m}$ - height of the cut material, $h_{p}$ - knife's reaching the material. 
The following constant input data have been used for calculations:

- width of the drum $l=0,8 \mathrm{~m}$,

- number of knives $z=8$ pcs.,

- turning angle of the knife's screw line $\varphi_{s}=9^{\circ}$,

- drum's angle $\alpha=20^{\circ}$,

- feeding speed of material to be cut $v=1,33 \mathrm{~m} \cdot \mathrm{s}^{-1}$,

- displacement of the crosscut edge towards the drum's axis $z_{m}=0,15 \mathrm{~m}$,

- unit cutting resistance $p=7000 \mathrm{~N} \cdot \mathrm{m}^{-1}$,

- drum's speed $n=1000 \mathrm{~min}^{-1}$.

During the analysis of the cutting process, 3 cases of cutting for different heights of material's cutting have been considered:

- case 1 for $h_{m l}=0,07 \mathrm{~m}$,

- case 2 for $h_{m 2}=0,12 \mathrm{~m}$,

- case 3 for $h_{m 3}=0,103 \mathrm{~m}$.

Additionally, each cutting case has been divided into phases. In case 1, the following phases have been detailed:

- knife's getting into material,

- correct material's cutting,

- knife's getting out.

In case 2:

- knife's getting into material,

- cutting with the whole knife's length,

- knife's getting out,

and in case 3 :

- knife's getting into material,

- knife's getting out from the material.

The cutting moment $M_{c}$ was calculated in accordance with the developed model for elementary cutting widths, for the parameters $\mathrm{x}_{\mathrm{A}}$ and $\mathrm{x}_{\mathrm{B}}$, which are the coordinates of the beginning and the end of the cutting edge for a given moment in accordance with the equation (1):

$$
M_{c}=2 \int_{x_{A}}^{x_{B}} p\left(r_{1}+x \operatorname{tg} \alpha\right) \sqrt{\left(y^{\prime}\right)^{2}+1} d x
$$

where:

$$
y^{\prime}=\frac{r_{1}+x \operatorname{tg} \alpha}{\sqrt{\left(r_{1}+x \operatorname{tg} \alpha\right)^{2}-z_{m}}} \operatorname{tg} \alpha
$$

The diagram of the cutting moment with its listed cases is presented in fig. 7 .

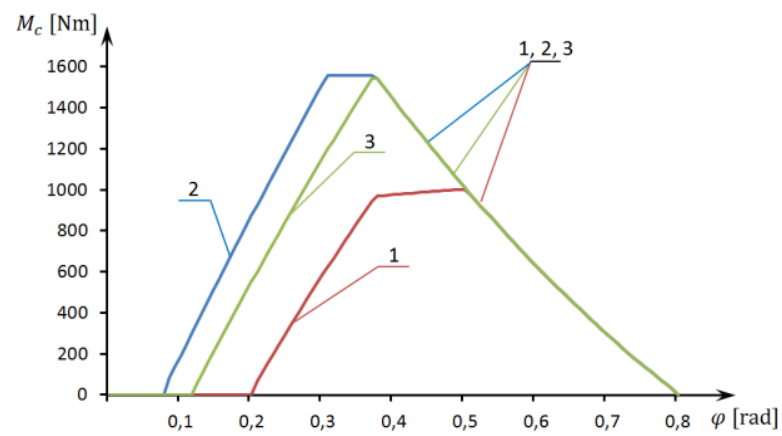

Fig. 7. Diagram of the cutting moment $M_{c}$ in the drum rotation's angle function $\varphi$ [own study]:1 - diagram for the case 1,2-diagram for the case 2,3-diagram for the case 3 .

There have also been conducted calculations for different constructions of a cutting drum (for different angles of the drum $\alpha$ ) at constant height of the material's layer. In fig. 8-10 there are presented the diagrams of the cutting moments' course $M_{c}$ (fig. 8), the medium cutting moment $M_{\dot{s} r}$ (fig. 9) and the cutting power $N_{c}$ (fig. 10) for a drum of conic type with screw knives for different angles of the drum $\alpha$ presented in tab. 1 .

Table 1. List of the input data changed during numerical

\begin{tabular}{|c|c|c|}
\hline \multirow{2}{*}{ Series No. } & \multirow{2}{*}{$\begin{array}{c}\text { Drum's angle } \\
\alpha\left[\ldots^{0}\right]\end{array}$} & $\begin{array}{l}\text { Minimum } \\
\text { radius } r_{1}[\mathrm{~m}]\end{array}$ \\
\hline & & $\begin{array}{c}\text { Maximum } \\
\text { radius } r_{2}[\mathrm{~m}]\end{array}$ \\
\hline \multirow{2}{*}{1} & \multirow{2}{*}{5} & 0,31 \\
\hline & & 0,34 \\
\hline \multirow{2}{*}{2} & \multirow{2}{*}{10} & 0,29 \\
\hline & & 0,36 \\
\hline \multirow{2}{*}{3} & \multirow{2}{*}{15} & 027 \\
\hline & & 0,38 \\
\hline \multirow{2}{*}{4} & \multirow{2}{*}{20} & 0,25 \\
\hline & & 0,40 \\
\hline \multirow{2}{*}{5} & \multirow{2}{*}{25} & 0,23 \\
\hline & & 0,42 \\
\hline
\end{tabular}
calculations for diagrams 8-10.

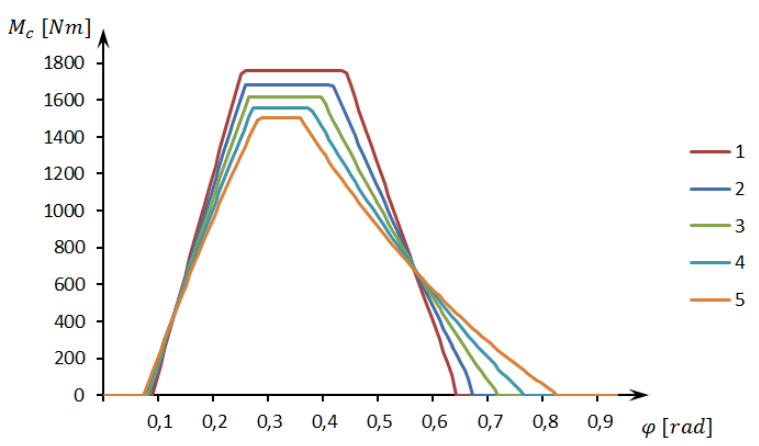

Fig. 8. Diagram of cutting moment $M_{c}$ in the function a drum's revolution $\alpha$ [own study]: $1-\alpha=5^{\circ}, 2-\alpha=10^{\circ}, 3-\alpha=15^{\circ}$, $4-\alpha=20^{\circ}, 5-\alpha=25^{\circ}$.

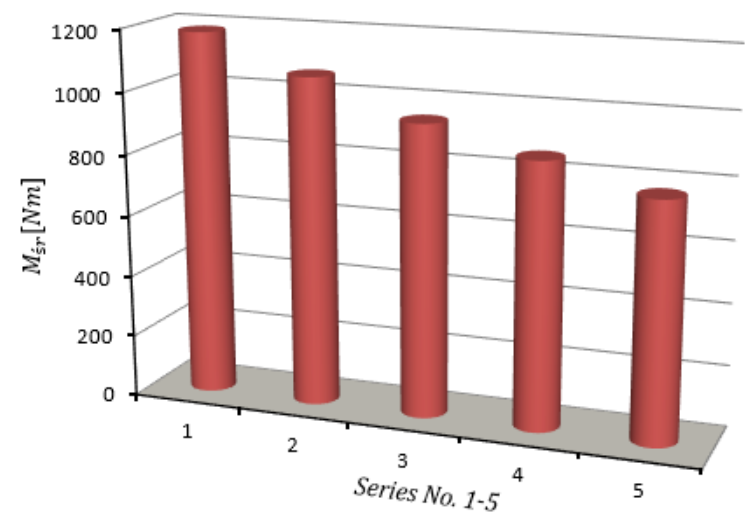

Fig. 9. Diagram of the average cutting moment $M_{\dot{s} r}$ of a cutting drum for different drum's angles $\alpha: 1-\alpha=5^{\circ}, 2-\alpha=10^{\circ}$, $3-\alpha=15^{\circ}, 4-\alpha=20^{\circ}, 5-\alpha=25^{\circ}$ [own study].

From the above presented diagram sit results, that together with the increase of the drum's angle $\alpha$, the values of the medium moment $M_{\dot{s}}$ and the cutting power $N_{c}$ decrease. The analysis was conducted for the angles 
from the range $\alpha=5-25^{\circ}$. That scope has been wellmatched because of the technological possibilities of the cutting process's realization.

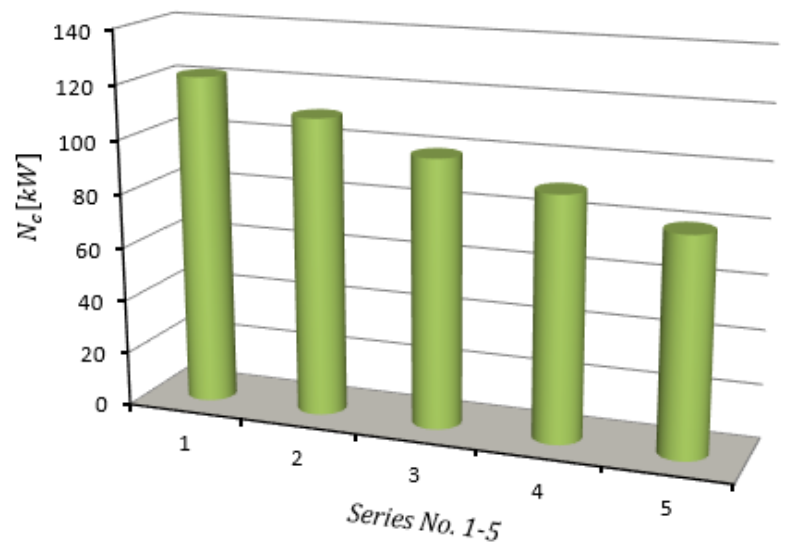

Fig. 10. Graph of cutting power $N_{c}$ of a cutting drum for different drum's angles $\alpha$ : $1-\alpha=5^{\circ}, 2-\alpha=10^{\circ}, 3-\alpha=15^{\circ}$, $4-\alpha=20^{\circ}, 5-\alpha=25^{\circ}$ [own study].

\section{Conclusions}

The exemplary results from simulation calculations conducted for the drum cutting assembly are presented in the article. These calculations have been conducted in the computer programme developed by the authors, on the created mathematical models. The originality of these calculations consists in the fact, that they have been conducted for real design and operating parameters of the drum cutting assemblies.

The conducted simulation calculations confirmed with the bench testing unambiguously show, that skew cutting conducted with the drum of conical construction is less energy consuming than the crosswise cutting conducted with a drum of a cylindrical construction.

\section{References}

1. A. Bochat, Theory and construction of cutting units of agricultural machines, in UTP University of Science and Technology, UTP Bydgoszcz, (2010)

2. M. Zastempowski, A. Bochat, Mathematical Modelling of Elastic Deflection of a Tubular CrossSection. Polish Maritime Research, 22(2) (2015)

3. P. Strzelecki, T. Tomaszewski, J. Sempruch, $A$ Method for Determining a Complete $S-N$ Curve Using Maximum Likelihood. Engineering Mechanics (2016)

4. B. Ligaj, G. Szala, Experimental verification of twoparametic models of fatigue characteristics by using the tests of S55J0 steel as an example. Polish Maritime Research, 17(1) (2010)

5. K. Migawa, L. Knopik, S. Wawrzyniak, Application of Genetic Algorithm to Control the Availability of Technical Systems. Engineering Mechanics (2016)

6. W. Keska, L. Gierz, Mathematical modeling and computer simulation of sowing. Conference: Agricultural Engineering: Land - Technik Ageng
2011. Solutions for Intelligent and Sustainable Farming, 2124 (2011)

7. M. Zastempowski, S. Borowski, J. Kaszkowiak, New Solutions in Harvesting Plants for Power Purposes. Trends in Agricultural Engineering (2013)

8. M. Zastempowski, A. Bochat, Modeling of Cutting Process by the Shear-Finger Cutting Block. Applied Engineering in Agriculture, 30(3) (2014)

9. M. Zastempowski, A. Bochat, Kinematics and dynamics of the drum cutting units. Springer Proceedings in Mathematics and Statistics (2016)

10. M. Karwowska, J. Mikolajczak, S. Borowski, Z. Dolatowski, J. Marc-Pienkowska, W. Budzinski, Effect of Noise Generated by the Wind Turbine on the Quality of Goose Muscles and Abdominal Fat. Annals of Animal Science, 14(2) (2014)

11. M. Zastempowski, A. Bochat, Innovative Constructions of Cutting and Grinding Assemblies of Agricultural Machinery. Proceeding of 6th International Conference on Trends in Agricultural Engineering (2016)

12. Claas advertising brochures. 